\title{
Influence of a magnetic field on the flow of a micropolar fluid sandwiched between two Newtonian fluid layers through a porous medium.
}

KUMAR YADAV, P., JAISWAL, S., ASIM, T. and MISHRA, R. 


\title{
Influence of magnetic field on the flow of micropolar fluid sandwiched between two Newtonian fluid layers through porous medium
}

\author{
Pramod Kumar Yadav ${ }^{* 1}$, Sneha Jaiswal ${ }^{2}$, Taimoor Asim ${ }^{3}$ and Rakesh Mishra ${ }^{4}$ \\ ${ }^{1,2}$ Department of Mathematics, Motilal Nehru National Institute of Technology Allahabad, \\ Allahabad-211004, India \\ ${ }^{3,4}$ School of Computing and Engineering, University of Huddersfield, Queensgate, \\ Huddersfield, U.K. (HD1 3DH)
}

\begin{abstract}
The present problem is concerned with the flow of micropolar/Eringen fluid sandwiched between two Newtonian fluid layers through the horizontal porous channel. The flow in both the regions is steady, incompressible and the fluids are immiscible. The flow is driven by a constant pressure gradient and a magnetic field of uniform strength is being applied in the direction perpendicular to the flow. The flow of electrically conducting fluids, in the three regions, is governed by the Brinkman equation with the assumption that the effective viscosity of each fluid is the same as the viscosity of the fluid. No-slip conditions at the end of the plates, continuity of velocity, continuity of shearing stress and constant rotational velocity at the interface have been used as the boundary conditions to get the solution of the problem considered. The numerical values of the solution obtained are used to analyse the effect of various transport parameters, such as permeability of porous region, magnetic number, viscosity ratio etc. on the velocity profile and micro rotational velocity profile graphically. Also, the variations in the flow rate and the wall shear stress, with respect to the governing parameters, are presented in tabular form.
\end{abstract}

Keywords: Micropolar fluid, porous medium, Hartmann Number, interface, flow rate.

\footnotetext{
* Corresponding author : Tel.: +91-9559022419

E-mail address: pramod547@gmail.com (Pramod Kumar Yadav).
} 


\section{Nomenclature}

$k \quad$ - dimensional permeability of the porous medium

$p \quad$-dimensional pressure

$x-y \quad$ - coordinates along the channel

$u_{i} \quad$-velocity in the $\mathrm{x}$-direction

$v_{i} \quad$-velocity in the y-direction

$\mu_{1} \& \mu \quad$-viscosity of the Newtonian fluid and micropolar fluid respectively

$\sigma_{1} \& \sigma \quad$-electrical conductivity of the Newtonian fluid and micropolar fluid respectively

K -material parameter

$\kappa \quad-$-vortex viscosity

$\gamma \quad-$ spin gradient viscosity

$m \quad$-viscosity ratio

$\alpha \quad$ - conductivity ratio

$i \quad$ - subscripts for region -I, region- II and region- III respectively. 


\section{Introduction}

In the present era, various research studies on fluid mechanics have solved many real-life problems including industrial, engineering, environmental etc., and more importantly, in the medical field. The study of bio-fluid mechanics emerges with significant applications in cardiovascular diseases. Due to the advance scope of the hydrodynamics, several problems related to magneto hydrodynamic (MHD) flows, transport in porous media, multiphase flows, channel flows etc., have been solved by a number of researchers with numerous applications. Recently, Yadav [1] has discussed the flow problem through the nonhomogeneous porous medium and also evaluated the drag force on the porous membrane. Oftentimes, it is observed in real-life that two or more immiscible fluid flows occur, such as flow of several immiscible oils through the bed of rocks or soils, flow in the rivers with several industrial fluids, blood flow in the arteries, releasing of dissolved gases from the crude oils into the reservoir rock etc. Few such works with various real-life applications were done by [2-4]. The presence of second fluid in different phase adds a number of complexities, due to interaction of transport phenomena of two different fluids and due to interfacial condition of two phases. Besides this, the flow of two immiscible fluids, either Newtonian-Newtonian or Newtonian-non Newtonian, has several important engineering and medical applications. One such type of problem is going to be discussed in this article.

It was found that Navier-Stokes equation is inadequate to describe the motion of the fluids exhibiting micro inertia, angular momentum, couple or non-symmetric stress. The classical laws of hydrodynamics fail to analyse the asymmetric deformation occurring in such types of fluids, such as liquid crystals, animal blood, biological fluids, muddy fluids, fluid with additives etc. The theory of micropolar fluids composing of rigid particles rotating in the viscous medium, was presented by Eringen [5,6]. Due to orientation of the particles in the medium, the micropolar fluids undergo both translational and rotational motion, which results in six degree of freedom. Many research studies have been carried out on micropolar fluids explaining various practical applications, for example, micropolar fluids can be used as lubricants as they have less friction coefficient than the Newtonian fluids [7, 8]. Many have discussed the flow of micropolar fluids compared to the flows of colloidal suspensions, liquid crystals, analysis with human and animals blood [9-12] and many more. Ariman et al. [12, 
13] gave a review on the micro-continuum approach and presented the applications of the fluids with the effects of micro-structures. Lukaszewicz [14] and Eringen [15] carried out a remarkable work by giving theories and applications of micropolar fluids.

Many investigations have been carried out on the flow characteristics of blood through the arteries. It was experimentally proved by some of the works that under different flow conditions, blood behaves sometimes as a Newtonian fluid [16-18] and sometimes as a nonNewtonian fluid $[19,20]$. These works have also showed that fluid (plasma) in the peripheral layer is Newtonian and in core layer (blood) is non-Newtonian. Fluid flow problems through porous channels have been studied by many researchers due to their use in the cardiovascular system. Ariman et al. [21] presented the micro-continuum approach of blood flow through rigid circular cylinders, by considering blood as a micropolar fluid. The conclusion made from the analytical solution obtained by Ariman et al. [21] was experimentally proved by Bugliarello and Sevilla [19] stating that micropolar fluid model is the best model for explaining the motion of microstructures in the blood flow. Due to clinical and physiological importance of micropolar fluids in the medical field, many problems have been solved with the assumption that blood behaves like a micropolar fluid. A two-fluid model of blood flow through the elastic cylindrical stenosed artery was considered by Ikbal et al. [22]. They considered Eringen's micropolar fluid flow in the core region and Newtonian fluid flow in the peripheral region. It was concluded that the resistance to flow and the shearing stress experienced by the walls of the arteries are higher in the case of two-phase model of blood, as compared to the single-phase Newtonian model. Chamkha et al. [23] investigated the problem of unsteady fully developed flow of two Newtonian immiscible fluids passing through a horizontal channel having permeable walls. The governing equations for the flow in both the regions have been solved using two-term harmonic and non-harmonic functions. Bakhtiyarov and Siginer [24] used opto mechanical method and experimentally proved the lubrication of non-Newtonian fluid by a Newtonian fluid, of two immiscible fluids flowing through a horizontal tube. They also discussed the importance of the results obtained in the transport of crude oil containing high wax, and in the designing of lubricated pipelines. Umavathi et al. [25] analytically solved the problem of flow through horizontal channel with the sandwiching of couple-stress fluid between two Newtonian fluids. They discussed the effects of various flow parameters and concluded that the couple-stress parameter promotes 
the flow. The problem of unsteady flow and heat transfer of the fluid through the horizontal channel comprising of porous medium between two Newtonian fluids has been solved by Umavathi et al. [26]. They discussed the influence of porous parameter, the frequency parameter etc. on the velocity and temperature profiles. Malashetty et al. [27] studied the effect of magnetic field and heat on the flow of two- immiscible fluids in the vertical channel. The non-linear governing equations are solved by regular perturbation method. Kumar et al. [28] discussed and solved the problem of flow of micropolar fluid and Newtonian fluid in the vertical channel under non-isothermal conditions.

The issues get severe on introducing the magnetic field to the flows, for example, MHD generators, pumps, nuclear reactors, filtration, and use in geothermal problems etc. The applications of magnetic field to the flow of immiscible fluids originate from reducing the flows in many medical and industrial purposes. Lohrasbi and Sahai [29] investigated twophase MHD flow and heat transfer in a horizontal channel. Malashetty and Leela [30] analysed the effect of Hartmann number on the flow of two-phase fluids passing through a horizontal channel. Malashetty and Umavathi [31] solved the problem of two-phase fluid flow and heat transfer under the effect of magnetic field, through the inclined channel. Chamkha [32] assumed the flow of two electrically conducting Newtonian fluids through porous and non-porous channels simultaneously.

Being motivated by above discussions and applications of immiscible MHD flows, here we have discussed the flow of micropolar fluid sandwiched between two Newtonian fluid regions. The fluids pass through the channel filled with porous medium having rigid walls and under the influence of magnetic field.

\section{Problem Statement and Governing Equations}

The mathematical model considered in the present work consists of a horizontal channel formed by two parallel plates extended infinitely in the $\mathrm{x}$ and $\mathrm{z}$ directions. The channel is filled with the porous medium of constant permeability. The flow of micropolar fluid in between the flow of Newtonian fluids is allowed to pass through the channel in such a way that the fluid interfaces are at equal width ' $h$ ' as shown in Fig. 1. 


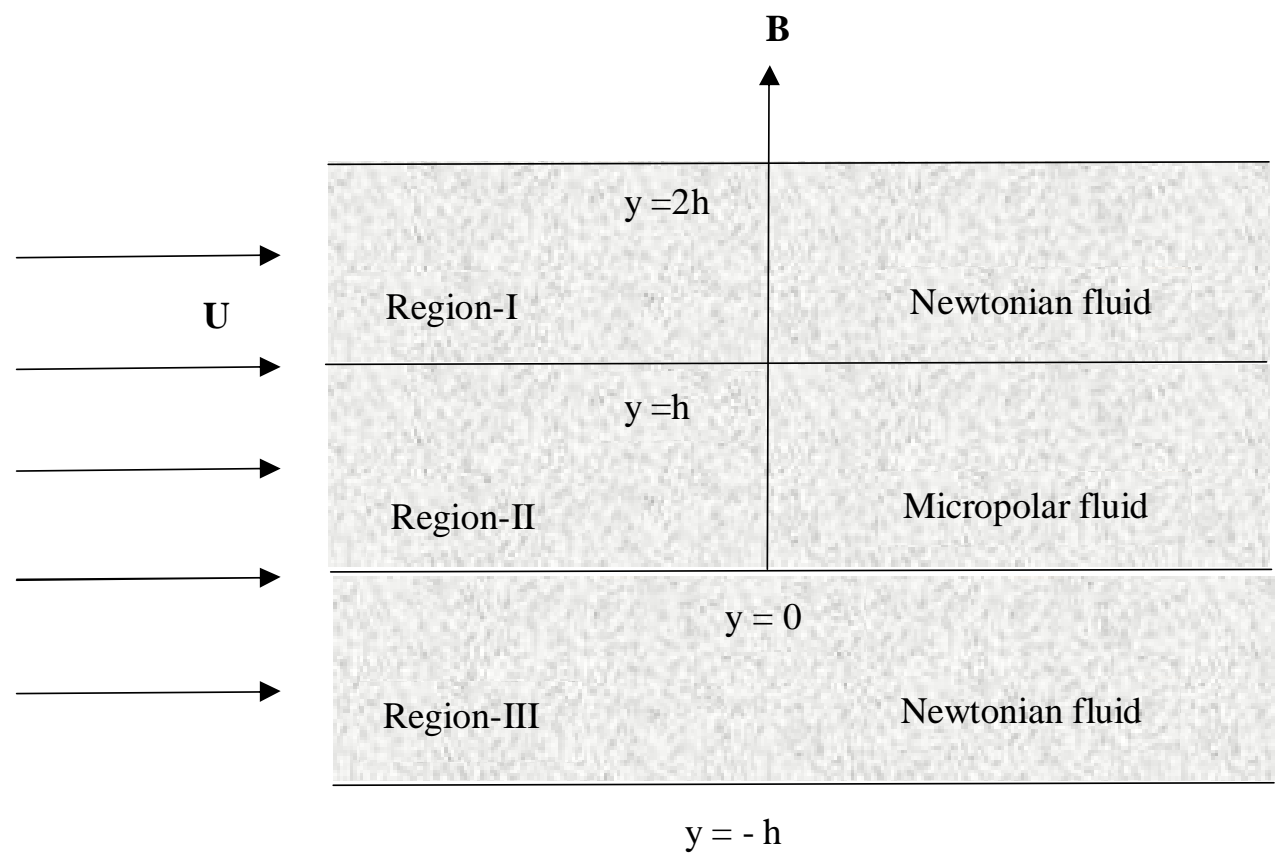

Fig. 1. Mathematical model of the problem

The steady, laminar, fully-developed and one-dimensional flow of fluids through the horizontal porous channel takes place in the presence of a transversal magnetic field. The flow in the channel is driven by a constant pressure gradient $\frac{\partial p}{\partial x}$. Let $u_{1}, u_{2}$ and $u_{3}$ be the flow velocities of fluids in the three regions along $\mathrm{x}$ direction. The Newtonian viscous fluids of same viscosity $\mu_{1}$ flow with different velocity $u_{1}$ and $u_{3}$ in the region-I and region-III respectively. The flow of micropolar fluid with velocity $u_{2}$ takes place in region-II. The magnetic field of uniform strength $B_{o}$ is applied perpendicular to the direction of the flow. Here, we also assume that the effective viscosity of the fluid is equal to the viscosity of the fluid.

Under the above discussed assumptions, the governing equations for the fluid flow through the horizontal porous channel are as follows: 
Region-I $(h \leq y \leq 2 h)$

$\mu_{1} \frac{d^{2} u_{1}}{d y^{2}}-\frac{\mu_{1}}{k} u_{1}-\sigma_{1} B_{0}^{2} u_{1}-\frac{d p}{d x}=0$

Region- II $(0 \leq y \leq h)$

$(\mu+\kappa) \frac{d^{2} u_{2}}{d y^{2}}+\kappa \frac{d \omega}{d y}-\frac{\mu}{k} u_{2}-\sigma B_{o}^{2} u_{2}-\frac{d p}{d x}=0$

$\gamma \frac{d^{2} \omega}{d y^{2}}-\kappa\left(2 \omega+\frac{d u_{2}}{d y}\right)=0$

Region-III $(-h \leq y \leq 0)$

$\mu_{1} \frac{d^{2} u_{3}}{d y^{2}}-\frac{\mu_{1}}{k} u_{3}-\sigma_{1} B_{0}^{2} u_{3}-\frac{d p}{d x}=0$.

Here $\sigma_{1}$ and $\sigma$ are the electrical conductivity of the Newtonian and micropolar fluids respectively. $\mu, \kappa$ and $\gamma$ are the viscosity, vortex viscosity and spin-gradient viscosity of the micropolar fluid respectively, and $\omega$ is the z-component of the micro rotational velocity of the micropolar fluid. The mathematical form for the spin-gradient viscosity $\gamma$ is $\gamma=\left(\mu+\frac{\kappa}{2}\right) j$ where $j$ is the micro inertia density.

\section{Solution of the Problem}

The solution of the problem considered in the present study, is discussed in detailed in the following sections.

\subsection{Non-Dimensional form of Governing Equations}

Equations (1) - (4) are transformed into dimensionless form by using the following nondimensional variables:

$u_{i}^{*}=\frac{u_{i}}{U}, y^{*}=\frac{y}{h}, x^{*}=\frac{x}{h}, p^{*}=\frac{p}{\mu_{1} U / h}, \omega^{*}=\frac{\omega}{U / h}$ and $k^{*}=\frac{k}{h^{2}}$. 
Here $U$ is the characteristic velocity and $h$ is the characteristic length. The micro-inertia density $\mathrm{j}$ is given by $j=h^{2}$.

Using equation (5), the dimensionless equations (dropping asterisk) for equations (1) - (4) can be written as:

Region-I $(0 \leq y \leq 2)$

$\frac{d^{2} u_{1}}{d y^{2}}-\left(n^{2}+M^{2}\right) u_{1}=\frac{d p}{d x}=P$

Region- II $(0 \leq y \leq 1)$

$(1+K) \frac{d^{2} u_{2}}{d y^{2}}+K \frac{d \omega}{d y}-\left(n^{2}+M_{1}^{2}\right) u_{2}=m \frac{d p}{d x}$,

$\left(1+\frac{K}{2}\right) \frac{d^{2} \omega}{d y^{2}}-K\left(2 \omega+\frac{d u_{2}}{d y}\right)=0$

Region-III $(-1 \leq y \leq 0)$

$\frac{d^{2} u_{3}}{d y^{2}}-\left(n^{2}+M^{2}\right) u_{3}=\frac{d p}{d x}=P$,

where $n=\frac{1}{\sqrt{k}}, M=B_{o} h \sqrt{\frac{\sigma_{1}}{\mu_{1}}}$ is the Hartmann number for the region-I and region-II, $K=\frac{\kappa}{\mu}$ is known as material parameter for the micropolar fluid and $M_{1}=M \sqrt{\frac{m}{\alpha}}, \quad m=\frac{\mu_{1}}{\mu}$ is viscosity ratio and $\alpha=\frac{\sigma_{1}}{\sigma}$ is conductivity ratio.

\subsection{Evaluation of flow velocity in all the three regions}

The solution for the flow velocity in the three regions can be obtained by direct method. Therefore, the velocities of the fluids for the respective regions are given as: 
Region-I $(0 \leq y \leq 2)$

$u_{1}(y)=C_{1} e^{A y}+C_{2} e^{-A y}-\frac{P}{A^{2}}$,

where $A^{2}=n^{2}+M^{2}$.

Region- $I I(0 \leq y \leq 1)$

$u_{2}(y)=C_{3} e^{\alpha_{1} y}+C_{4} e^{-\alpha_{1} y}+C_{5} e^{\beta_{1} y}+C_{6} e^{-\beta_{1} y}-\frac{m}{n^{2}+M_{1}^{2}} P$,

$\omega(y)=-\eta\left(C_{3} e^{\alpha_{1} y}-C_{4} e^{-\alpha_{1} y}\right)-\xi\left(C_{5} e^{\beta_{1} y}-C_{6} e^{-\beta_{1} y}\right)$,

where

$$
\begin{aligned}
& \alpha_{1}=\sqrt{\frac{\alpha^{2}+\sqrt{\alpha^{4}-4 \beta^{2}}}{2}}, \beta_{1}=\sqrt{\frac{\alpha^{2}-\sqrt{\alpha^{4}-4 \beta^{2}}}{2}}, \\
& \alpha^{2}=\frac{2 K}{(1+K)}+\frac{n^{2}+M_{1}^{2}}{(1+K)}, \beta^{2}=\frac{4 K\left(n^{2}+M_{1}^{2}\right)}{(2+K)(1+K)}, \\
& \eta=\frac{\alpha_{1}^{3}}{4 K^{2}}(1+K)(2+K)+\frac{\alpha_{1}}{2}-\frac{\alpha_{1}}{4 K^{2}}\left(n^{2}+M_{1}^{2}\right)(2+K), \\
& \xi=\frac{\beta_{1}^{3}}{4 K^{2}}(1+K)(2+K)+\frac{\beta_{1}}{2}-\frac{\beta_{1}}{4 K^{2}}\left(n^{2}+M_{1}^{2}\right)(2+K) .
\end{aligned}
$$

Region-III $(-1 \leq y \leq 0)$

$u_{3}(y)=C_{7} e^{A y}+C_{8} e^{-A y}-\frac{P}{A^{2}}$.

\subsection{Evaluation of flow rate in the porous channel}

The non- dimensional volumetric flow rate of the fluid through the horizontal porous region is evaluated as:

$$
Q=\int_{-1}^{0} u_{3} d y+\int_{0}^{1} u_{2} d y+\int_{1}^{2} u_{1} d y
$$


Using the values from (10), (11) and (13) in equation (14), we can get the flow rate as:

$$
\begin{aligned}
Q= & \frac{C_{1} e^{A}}{A}\left(e^{A}-1\right)-\frac{C_{2} e^{-A}}{A}\left(e^{-A}-1\right)+\frac{C_{3}}{\alpha_{1}}\left(e^{\alpha_{1}}-1\right)-\frac{C_{4}}{\alpha_{1}}\left(e^{\alpha_{1}}-1\right)+\frac{C_{5}}{\beta_{1}}\left(e^{\beta_{1}}-1\right)- \\
& \frac{C_{6}}{\beta_{1}}\left(e^{-\beta_{1}}-1\right) \frac{C_{7} e^{-A}}{A}\left(e^{A}-1\right)-\frac{C_{8} e^{A}}{A}\left(e^{-A}-1\right)
\end{aligned}
$$

\subsection{Evaluation of the wall shear stress}

Here, an attempt has been made to calculate the skin friction on the top and bottom of the channel in order to analyse the effects of different fluid parameters.

The wall shear stresses on top and bottom of the horizontal porous channel i.e. $\tau_{T}$ and $\tau_{B}$ respectively, in dimensionless form are given by the following:

$$
\tau_{T}=\left(\frac{d u_{1}}{d y}\right)_{y=2} \text { and } \quad \tau_{B}=\left(\frac{d u_{3}}{d y}\right)_{y=-1}
$$

Using equations (10), (13) and (16), we get the values of skin frictions involving arbitrary constants:

$$
\begin{aligned}
& \tau_{T}=A\left(C_{1} e^{2 A}-C_{2} e^{-2 A}\right), \\
& \tau_{B}=A\left(C_{7} e^{-A}-C_{8} e^{A}\right) .
\end{aligned}
$$

The arbitrary constants $C_{1}, C_{2}, C_{3}, C_{4}, C_{5}, C_{6}, C_{7}$ and $C_{8}$ have been evaluated using the boundary and interface conditions.

\section{Boundary and Interface conditions}

In order to get the solution of the concerned problem i.e. to obtain the values of eight arbitrary constants, we need mathematically consistent boundary and interface conditions. Since all the four equations are second order differential equations, therefore, eight conditions are needed to solve the problem. The non-dimensional forms of the boundary and interface conditions are described below. 


\subsection{No-Slip boundary conditions}

The observations, based on the experiments on the fluid flow through the solid surface, indicate that the fluid in motion stops completely at a solid boundary and is considered to have zero velocity. The direct contact of the fluid particles with solid surface results in sticking of fluid to the boundary and hence, there is no slip at the solid surface. This is known as no-slip condition.

The first two boundary conditions are derived from the fact that there is no flow at the rigid boundaries of the porous horizontal channel i.e.:

$u_{1}=0$ and $u_{3}=0$ at $y=2$ and $y=-1$ respectively.

\subsection{Interface conditions}

The continuity of velocity, continuity of stresses and constant cell rotational velocity at the interfaces are used as the interface conditions for solving the problem i.e.:

$u_{1}=u_{2}$ at $y=1$ and $u_{2}=u_{3}$ at $y=0$,

$\mu_{1} \frac{d u_{1}}{d y}=(\mu+\kappa) \frac{d u_{2}}{d y}+\kappa \omega$ at $y=1$ and $(\mu+\kappa) \frac{d u_{2}}{d y}+\kappa \omega=\mu_{1} \frac{d u_{3}}{d y}$ at $y=0$,

$\frac{d \omega}{d y}=0$ at both the interfaces i.e. $y=1$ and $y=0$.

Using aforementioned boundary and interface conditions in equations (10) - (13), we get the system of linear algebraic equations involving the arbitrary constants. The system of linear equations obtained are as follows:

$$
\begin{aligned}
& C_{1} e^{2 A}+C_{2} e^{-2 A}=\frac{P}{A^{2}}, \\
& C_{7} e^{-A}+C_{8} e^{A}=\frac{P}{A^{2}}, \\
& C_{1} e^{A}+C_{2} e^{-A}-C_{3} e^{\alpha_{1}}-C_{4} e^{-\alpha_{1}}-C_{5} e^{\beta_{1}}-C_{6} e^{-\beta_{1}}=\frac{P}{A^{2}}-\frac{m P}{M_{1}^{2}+n^{2}},
\end{aligned}
$$




$$
\begin{aligned}
& C_{3}+C_{4}+C_{5}+C_{6}-C_{7}-C_{8}=\frac{m P}{M_{1}^{2}+n^{2}}-\frac{P}{A^{2}}, \\
& C_{1} A m e^{A}-C_{2} A m e^{-A}-C_{3}\left(\alpha_{1}(1+K)+K \eta\right) e^{\alpha_{1}}+C_{4}\left(\alpha_{1}(1+K)+K \eta\right) e^{-\alpha_{1}} \\
& -\left(\beta_{1}(1+K)+K \xi\right) C_{5} e^{\beta_{1}}+C_{6}\left(\beta_{1}(1+K)+K \xi\right) e^{-\beta_{1}}=0, \\
& C_{3}\left(\alpha_{1}(1+K)+K \eta\right)-C_{4}\left(\alpha_{1}(1+K)+K \eta\right)+C_{5}\left(\beta_{1}(1+K)+K \xi\right) \\
& -C_{6}\left(\beta_{1}(1+K)+K \xi\right)-C_{7} A m+C_{8} A m=0, \\
& C_{3} \alpha_{1} \eta+C_{4} \alpha_{1} \eta+C_{5} \beta_{1} \xi+C_{6} \beta_{1} \xi=0, \\
& C_{3} \alpha_{1} \eta e^{\alpha_{1}}+C_{4} \alpha_{1} \eta e^{-\alpha_{1}}-C_{5} \beta_{1} \xi e^{\beta_{1}}+C_{6} \beta_{1} \xi e^{-\beta_{1}}=0 .
\end{aligned}
$$

By the use of MATHEMATICA 10.3, we were able to evaluate the values of constants.

\section{Results and Discussions}

A detailed discussion on the results obtained is presented in the following sections.

\subsection{Effect of different transport properties on the flow velocity}

Critical analyses of the effect of different transport properties, such as material parameter, viscosity ratio, magnetic field, conductivity ratio and permeability, on the flow velocity are have been presented in this section.

\subsubsection{Effect of material parameter}

Figs. 2-3 show the effects of material parameter on the flow velocity of the fluids in the three respective porous regions when, permeability $k=1.1$, viscosity ratio $m=1$, pressure gradient $P=-0.7$ magnetic number $M=1.1$ and conductivity ratio $\alpha=1$. It has been observed that as the value of material parameter of micropolar fluid increases, the flow velocity in region-II decreases (Fig. 3). For higher values of material parameter K, the feature of graph changes from parabolic to straight. However, increase in material parameter value doesn't affect the flow of Newtonian fluids in regions I and III. This is because material parameter is a property of micropolar fluid only, which usually describes the micro rotational property of the fluid. In fig.2, as the value of material parameter $\mathrm{K}$ increases from 0.01 to 2 , the flow of micropolar fluid in region II decreases slowly, and for $\mathrm{K}>2$ the variation seems to be the same i.e. the variation in velocity (micropolar fluid) can only be observed for $0<K \leq 2$. 


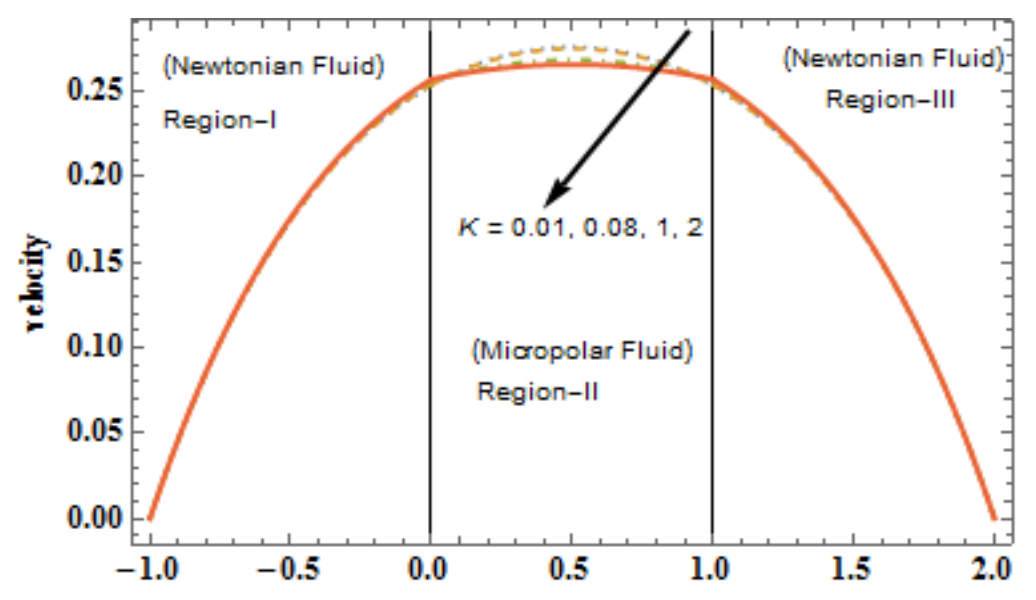

Fig. 2 Variation of velocity with material parameter K

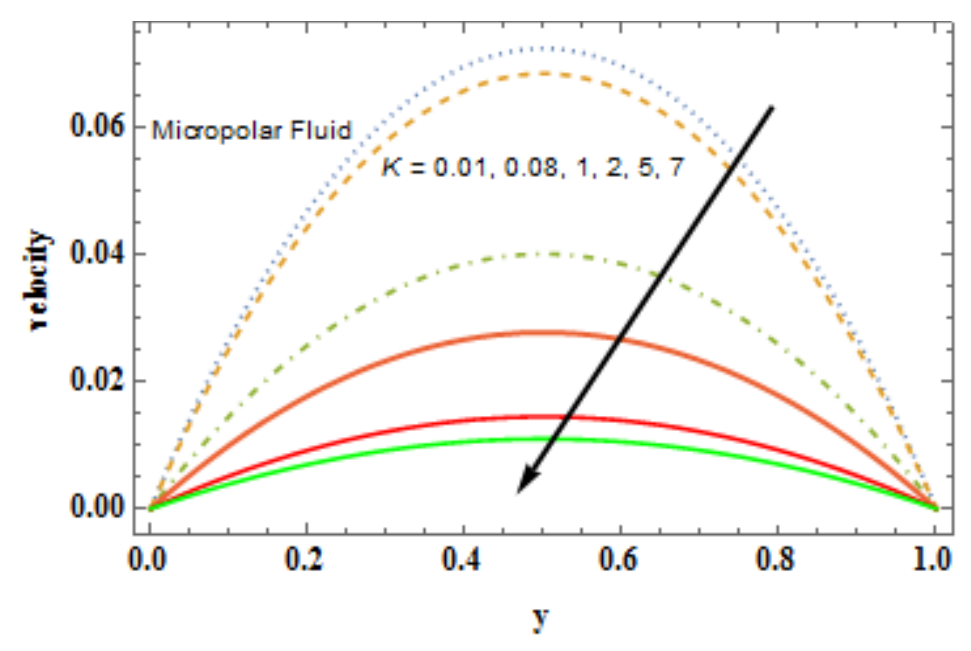

Fig. 3 Variation of micropolar fluid velocity with the material parameter

Fig. 3 represents the variation of micropolar fluid velocity with material parameter, when only the micropolar fluid is allowed to flow through the porous horizontal channel. It has been noticed that the material parameter affects the flow velocity even when it is higher than 2. As the value increases from $K=7$ onwards, the curves tend to be straight lines. 


\subsubsection{Effect of viscosity ratio}

Fig. 4 depicts the effects of viscosity ratio on the flow velocity when $k=1.1, K=2$, $P=-0.7, M=1.1, \alpha=1$. It can be observed that the increasing values of viscosity ratio promote the flows of the fluids in all the three regions of the horizontal porous channel. When $m=0.1$, the flow in regions I and III is more than the flow of micropolar fluid in region-II, while when $m=3$, the flow in region-II is more than the flow of Newtonian viscous fluids in regions I and III.

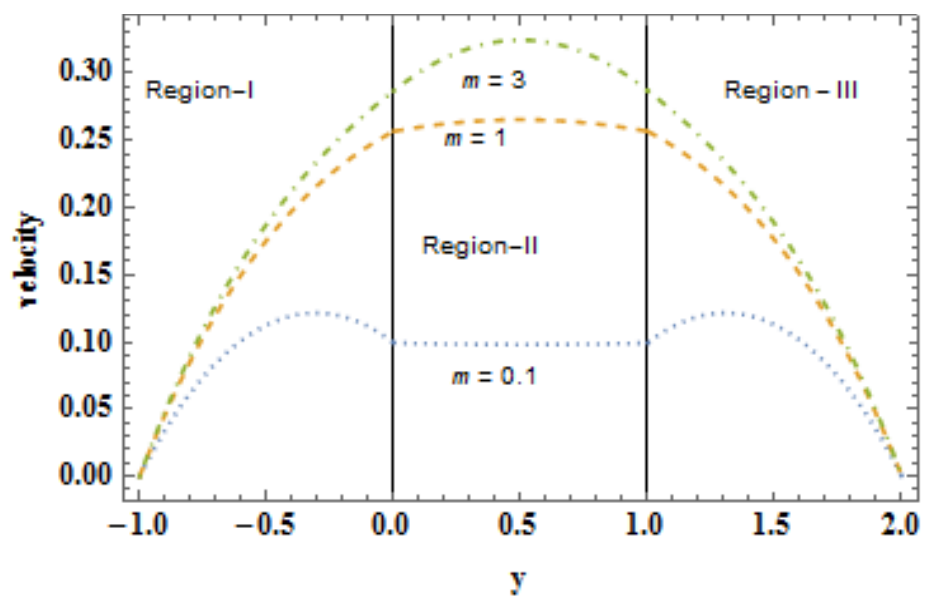

Fig. 4 Variation of flow velocity with the viscosity ratio

\subsubsection{Effect of magnetic field}

Fig. 5 depicts the effects of the strength of magnetic field on the flow velocity when $k=1.1$, $K=2, P=-0.7, m=1, \alpha=1$. It is observed that the flow velocity in the porous channel decreases as the strength of magnetic field i.e. the value of Hartmann number increases. This is due to increasing resistive force (Lorentz force) associated with the applied magnetic field. Hence, reducing the strength of applied magnetic field can increase the blood flow in the porous arteries, while increasing the values of Hartmann number can slow the flow through the porous region. 


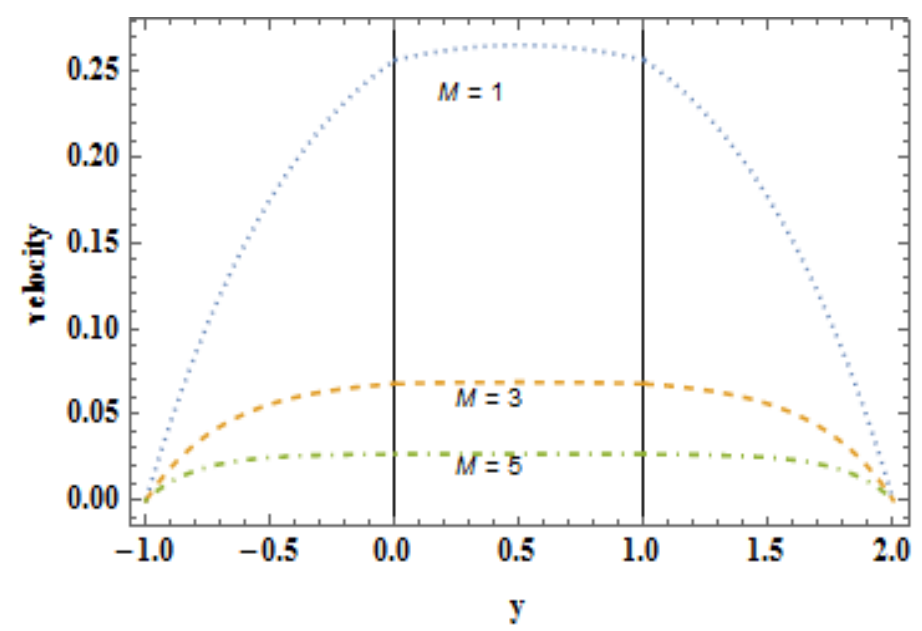

Fig. 5 Variation of flow velocity with the Hartmann Number

\subsubsection{Effect of conductivity ratio}

Fig. 6 shows the effect of conductivity ratio on the flow velocity when $k=1.1, K=2$, $P=-0.7, m=1, M=1$. It can be noticed that increasing the conductivity ratio increases the flow in all the three regions of the porous channel. When electrical conductivity of the micropolar fluid is less than that of the Newtonian fluid, the flows in regions I and III are more than in region-II.

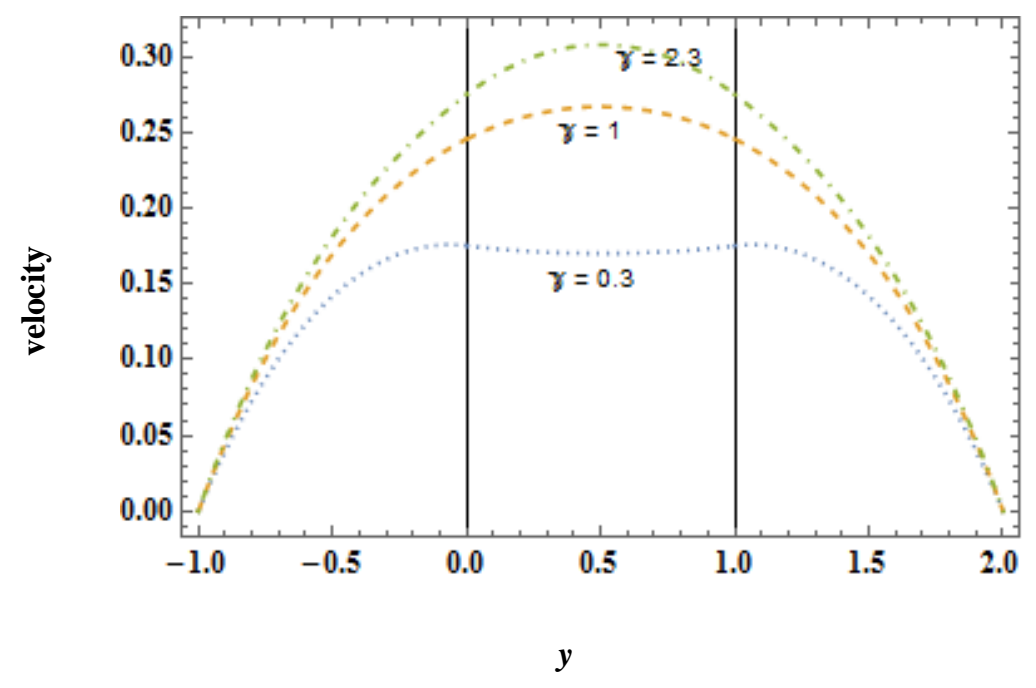

Fig. 6 Variation of flow velocity with the conductivity ratio 


\subsubsection{Effect of permeability}

The effects of permeability of the porous medium on the flow velocity of the fluids, in their respective regions, are shown in Fig. 7 when $\alpha=1, K=2, P=-0.7, m=1, M=1$.

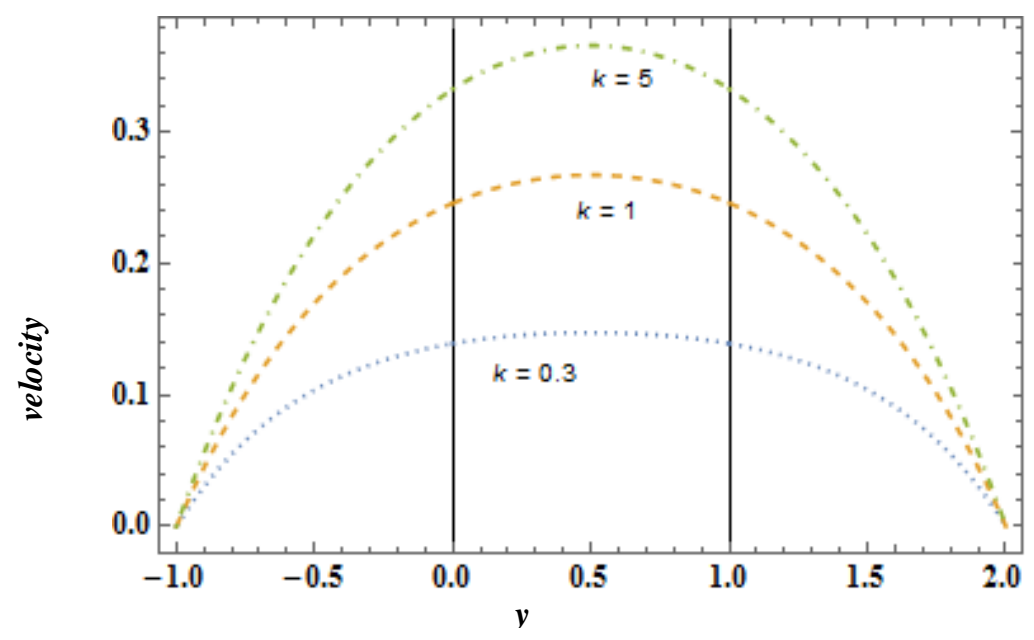

Fig. 7 Variation of flow velocity with the permeability

The permeability of the porous media is defined as the capability of porous media to pass or transport the fluids through it. It can be clearly seen from fig.7 that as the permeaility of the porous region in the horizontal channel increases, it results in the increase of fluid flow in all the three regions. As the permeability increases to higher values, the flow also increases to the same extent.

\subsection{Effect of different transport properties on the micro rotational velocity}

A detailed analysis of the effect of different transport properties on the micro rotational velocity has been presented in this section.

\subsubsection{Effect of material parameter}

The effect of material parameter on the micro rotational velocity of the micropolar fluid in region-II is shown in Fig. 8 when $\alpha=1, k=0.1, P=-7.0, m=1, M=1.5$. It is clear from fig. 8 that above $y=0.5$ the micro rotation velocity increases with material parameter, and below $y=0.5$, it decreases with increase in the value of the material parameter. 


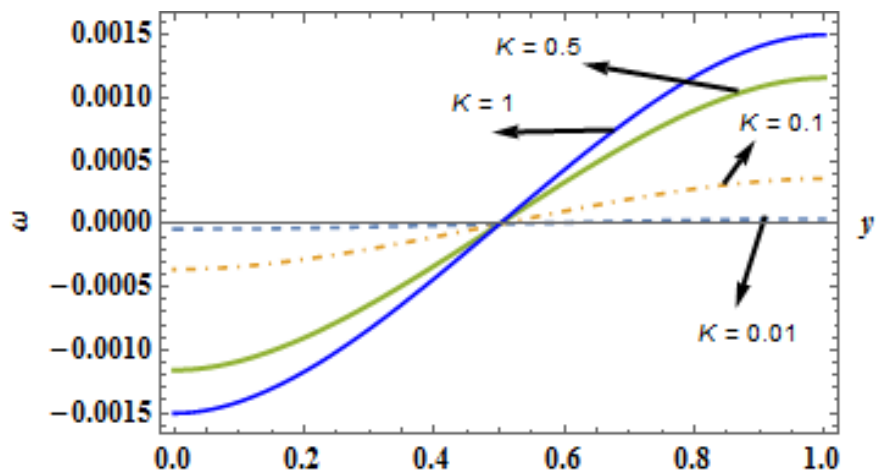

Fig. 8 Variation of micro rotational velocity with the material parameter

\subsubsection{Effect of viscosity ratio}

The effect of viscosity ratio on the micro rotational velocity of the micropolar fluid in region-II is illustrated in Fig. 9 when $\alpha=1, K=1, P=-7.0, k=0.1, M=1.5$. It can be noticed that when $m<1$, the micro rotational velocity increases with increase in viscosity ratio for $0.5>\mathrm{y}$, however, above $y=0.5$, it decreases.

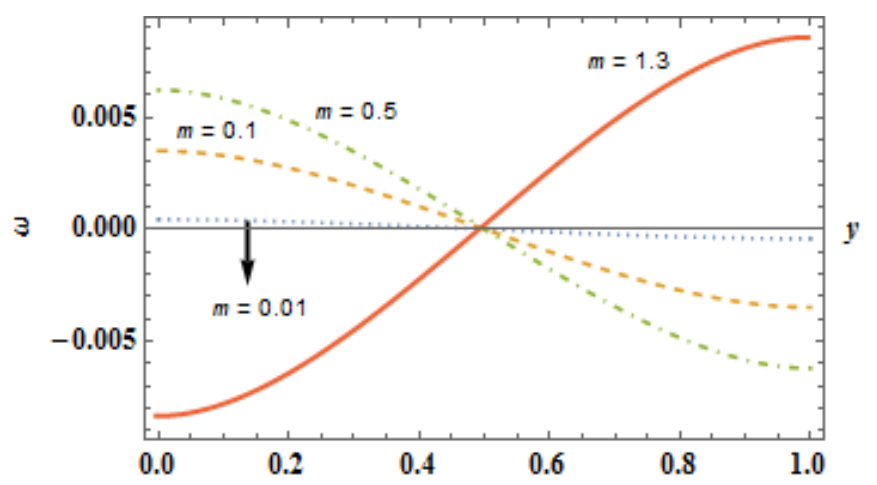

Fig. 9 Variation of micro rotational velocity with viscosity

\subsubsection{Effect of Hartmann number}

The effect of applied magnetic field on the micro rotational velocity of the micropolar fluid in region-II is analyzed in Fig. 10 when $\alpha=1, K=1, P=-0.7, m=1, k=1.1$. It can be seen that above $y=0.5$, as the strength of magnetic field increases, the micro rotational 
velocity decreases rapidly, but it never reached zero. Below $y=0.5$, the micro rotational velocity increases with increase in Hartmann number.

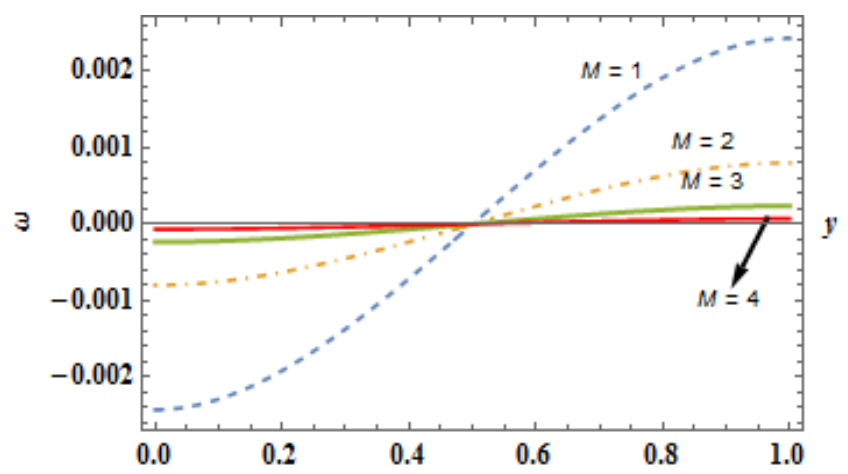

Fig. 10 Variation of micro rotational velocity with Hartmann number

\subsubsection{Effect of conductivity ratio}

The effects of conductivity ratio on the micro rotational velocity of the micropolar fluid in region-II is analyzed in Fig. 11 when $M=1.5, K=1, P=-7.0, m=1, k=0.1$.

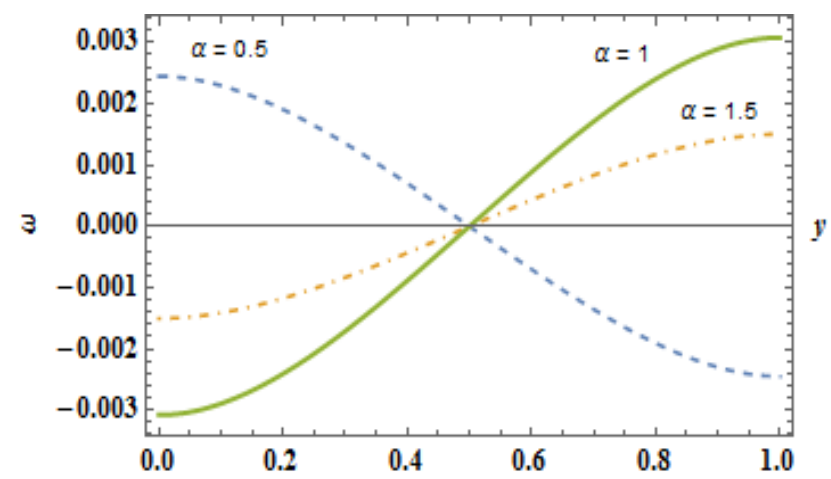

Fig. 11 Variation of micro rotational velocity with conductivity ratio

The variations of micro rotational velocity with conductivity ratio below and above $y=0.5$ can be observed in fig.11. Below $y=0.5$, it has been observed that for lower value of conductivity ratio, the micro rotational velocity is higher. And, above $y=0.5$, the micro rotational velocity attains minimum value for lower values of conductivity ratio. 
5.3 Effect of different transport properties on the volumetric flow rate and wall shear stress

The effect of various flow parameters like magnetic number $(0<M<8)$, permeability $(0<k<8))$, viscosity ratio $(0<m<8)$, material parameter $(0<K<3)$ and conductivity ratio $(0<\alpha<4)$ with pressure difference $(P=-0.7)$ on the flow rate and shear stress at the top and bottom of the channel has been critically analyzed in this section. The results obtained have been presented in tabular form here (Table 1).

Table 1

\begin{tabular}{|l|c|c|c|c|}
\hline Parameters & Values & $\mathbf{Q}$ & $\boldsymbol{\tau}_{\mathbf{T}}$ & $\boldsymbol{\tau}_{\mathbf{B}}$ \\
\hline \multirow{4}{*}{ Magnetic Number } & & & -0.4828 & 0.4828 \\
\cline { 2 - 5 } & 3 & 0.5831 & -0.2214 & 0.2214 \\
\cline { 2 - 5 } & 5 & 0.1670 & -0.1373 & 0.1373 \\
\hline \multirow{4}{*}{ Permeability } & 7 & 0.0704 & -0.0990 & 0.0990 \\
\cline { 2 - 5 } & 0.3 & 0.0381 & -0.3356 & 0.3356 \\
\cline { 2 - 5 } & 1 & 0.3296 & -0.4848 & 0.4848 \\
\cline { 2 - 5 } Viscosity ratio & 5 & 0.5665 & -0.5956 & 0.5956 \\
\cline { 2 - 5 } & 0.1 & 0.7559 & -0.6065 & 0.6065 \\
\cline { 2 - 5 } & 1 & 0.7745 & -0.3692 & 0.3692 \\
\cline { 2 - 5 } & 3 & 0.2827 & -0.4828 & 0.4828 \\
\hline \multirow{3}{*}{ Material Parameter } & 0.5831 & -0.5046 & 0.5046 \\
\cline { 2 - 5 } & 0.08 & 0.6589 & -0.5058 & 0.5058 \\
\cline { 2 - 5 } & 1 & 0.6840 & -0.4812 & 0.4810 \\
\hline \multirow{3}{*}{ Conductivity Ratio } & 2 & 0.5858 & -0.4828 & 0.4812 \\
\cline { 2 - 5 } & 0.3 & 0.5855 & -0.4835 & 0.4828 \\
\cline { 2 - 5 } & 1 & 0.5838 & -0.4828 & 0.4835 \\
\cline { 2 - 5 } & 2.3 & 0.4286 & -0.5048 & 0.48289 \\
\hline
\end{tabular}

The variations in the flow rate with different governing parameters also validate the graphical results of the flow velocity. It can be evaluated from table 1 that flow rate in the horizontal porous channel, in the presence of transversal magnetic field, increases with increase in the permeability of the porous medium, viscosity ratio and conductivity ratio. 
The shear stress acting on the top and bottom of the porous channel increases (in magnitude) with increase in the values of permeability, viscosity ratio, material parameter and conductivity ratio. However, the wall shear stress on the both wall of the porous horizontal channel decreases with increase in the strength of the applied magnetic field.

\section{Conclusions}

The problem of flow of micropolar fluid sandwiched between two Newtonian fluids through a horizontal porous channel, under the effect of transverse magnetic field, has been solved analytically. Analytical expressions for the flow velocity, micro rotational velocity of the micropolar fluid, flow rate and wall shear stress have been obtained using appropriate boundary and interface conditions. It has been found out that the viscosity ratio, conductivity ratio and the permeability of the porous media promotes the flows' velocities in the porous channel whereas, the material parameter of the micropolar fluid, and the Hartmann number, suppresses the flows. The tabulated variations observed in the wall shear stress have also been analyzed, and it has been found out that the stress applied by the flows on the upper and lower layer of the porous channel can be reduced by applying the magnetic field in the transverse direction of the flow.

Acknowledgment: The first Author is thankful to SERB, New Delhi for supporting this research work under the research grant SR/FTP/ MS-47/ 2012.

\section{References}

[1] P.K. Yadav, The Eur. Phys.J. Plus 133, 1 (2018).

[2] I. Ansari, S. Deo., Natl. Acad. Sci. Lett. 40, 211 (2017).

[3] P.K.Yadav, S. Jaiswal, B.D. Sharma, Appl. Math. Mech. (Eng. Ed.) (Accepted), (2018).

[4] P.K.Yadav, S. Jaiswal, Cand. J. Phy. (Accepted), (2018).

[5] A.C. Eringen, Int. J. Eng. Sci. 2, 205 (1964).

[6] A.C. Eringen, J. Math. Mech. 16, 1 (1966).

[7] M.M. Khonsari, D.E. Brewe, ASLE Tribology Trans. 32, 155 (1989).

[8] M.M. Khonsari, Acta Mech. 81, 235 (1990).

[9] H. Busuke, T. Tatsuo, Int.J. Eng. Sci. 7, 515 (1969).

[10] J.D. Lee, A.C. Eringen, The J. Chem. Phy. 55, 4509 (1971).

[11] F.E. Lockwood, M.T. Benchaita, S.E. Friberg, ASLE transactions 30, 539 (1986). 
[12] T. Ariman, M.A. Turk, N.D. Sylvester, Int.J. Eng. Sci. 11, 905 (1973).

[13] T. Ariman, M.A. Turk, N.D. Sylvester, Int. J. Eng. Sci. 12, 273 (1974).

[14] G. Lukaszewicz, Springer Science \& Business Media, (1999).

[15] A.C. Eringen, Springer Science \& Business Media, (2001).

[16] L. Bayliss, In Deformation and Flow in Biological Systems, ed. FREY-WISSLING, A., Amsterdam: North Holland Publishing Co. 354 (1952).

[17] Y.C. Fung, Federation Proceedings. 25, 1761 (1966).

[18] H.S. Lew, Y.C. Fung, J. Biomech. 3, 23 (1970).

[19] G. Bugliarello, J. Sevilla, Biorheology. 7, 85 (1970).

[20] H.L. Goldsmith, R. Skalak, Annual Review of Fluid Mechanics. 7, 213 (1975).

[21] T. Ariman, M.A. Turk, N.D. Sylvester, J. Appl. Mech. 41, 1 (1974).

[22] M. A. Ikbal, S. Chakravarty, P.K. Mandal, Comput. Math. Appl. 58, 1328 (2009).

[23] A.J. Chamkha, J.C. Umavathi, A. Mateen, Int. J. Fluid Mech. Res. 31, 13 (2004).

[24] S.I. Bakhtiyarov, D.A. Siginer, ICHMT Digital Library Online. Begel House Inc., 1997.

[25] J.C. Umavathi, A.J. Chamkha, M.H. Manjula, A. Al-Mudhaf, Canadian J. Phy. 83, 705 (2005).

[26] J.C. Umavathi, I.C. Liu, J. Prathap-Kumar, D. Shaik-Meera, Appl. Math. Mech. - Engl. Ed. 31, 1497 (2010).

[27] M.S. Malashetty, J C Umavathi , J Prathap Kumar, Heat and Mass Transfer 42, 977 (2006).

[28] J.P. Kumar, J.C. Umavathi, A.J. Chamkha, I. Pop, Appl. Math. Model. 34, 1175 (2010).

[29] J. Lohrasbi, V. Sahai, Appl. Sci. Res. 45, 53 (1988).

[30] M.S. Malashetty, V. Leela, Int. J. Eng. Sci. 30, 371 (1992).

[31] M.S. Malashetty, J.C. Umavathi, Int. J. Multiphase Flow. 23, 545 (1997).

[32] A.J. Chamkha, J. Fluids Eng. 122, 117 (2000). 